International Journal of Pure and Applied Mathematics

Volume 103 No. 3 2015, 547-556

ISSN: 1311-8080 (printed version); ISSN: 1314-3395 (on-line version)

url: http://www.ijpam.eu

doi: http://dx.doi.org/10.12732/ijpam.v103i3.15

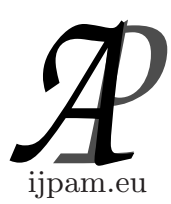

\title{
THE PROBABILISTIC HAUSDORFF QUASI-METRIC AND RIGHT K-COMPLETENESS
}

\author{
Abderrahim Mbarki ${ }^{1} \S$, Abedelmalek Ouahab ${ }^{2}$, Rachid Naciri ${ }^{3}$ \\ ${ }^{1}$ National School of Applied Sciences \\ P.O. Box 669, Oujda University \\ Matsi Laboratory, MOROCCO \\ ${ }^{2}$ Department of Mathematics \\ Oujda University \\ 60000, Oujda Morocco, Matsi Laboratory, NOROCCO \\ ${ }^{3}$ Matsi Laboratory \\ Oujda University \\ 60000, Oujda, MOROCCO
}

\begin{abstract}
By considering probabilistic quasi-(pseudo-)metric spaces, we introduce and study the concept of probabilistic Hausdorff quasi-(pseudo-)metric metric that extend the corresponding notions of probabilistic Hausdorff metric. Finally completeness property of this notion are explored.
\end{abstract}

AMS Subject Classification: 54E70, 54A40, 54B20

Key Words: probabilistic quasi-metric space, probabilistic Hausdor quasimetric, right K-completeness

\section{Introduction}

Throughout this paper for a topological space $(X, \mathcal{T})$; we mean by $\mathcal{P}_{0}(X)$ the collection of non-empty subsets. If $A$ is a subset of $X$, we will denote by $c l_{\mathcal{T}}(A)$, the closure of $A$ in $X$.

Received: May 20, 2015

(C) 2015 Academic Publications, Ltd.

${ }^{\S}$ Correspondence author url: www.acadpubl.eu 
The concept of a probabilistic metric spaces is a generalization of that of a metric spaces. The origin of the theory dates back to a paper published by K. Menger in 1942 [7] in which the distance $d(x, y)$ between two point $x, y$ was replaced by a real function $F_{x y}$ whose value $F_{x y}(t)$, for any positive real $t$, is interpreted as the probability that the distance between $x$ and $y$ is less than or equal to $t$. The study of these spaces was performed extensively by B.Schweizer and A.Sklar ([9]).

In 1968 R.J. Egbert [3] extended the classical Hausdorff distance to the probabilistic setting and introduced the notion of probabilistic Hausdorff distance in Menger space, soon Tardiff [10] enlarged this notion to the case of general probabilistic metric spaces (see also [9]). Thereafter, many works have for object the examination of the conditions under which the completeness property possessed by the probabilistic metric can be preserved by its hyperspaces. In the sequel to such developments, we introduce and study the notion of the probabilistic Hausdorff quasi-metric of a given probabilistic quasi-metric space that generalize the corresponding notion of the probabilistic Hausdorff metric.

Terms and undefined concepts about quasi-uniform spaces and quasi-pseudometric spaces may be found in [4] and [5]

Recall that a quasi-pseudo-metric on a nonempty set $X$ is a nonnegative real valued function defined on $X \times X$ such that for all $x, y, z \in X$ we have

i. $d(x, x)=0$,

ii. $d(x, y) \leq d(x, z)+d(z, y)$.

If $d$ satisfies conditions $(i)$ and $(i i)$ above but we allow $d(x, y)=+\infty$, then $d$ is said to be an extended quasi-pseudo-metric on $X$. If $d$ is quasi-pseudo-metric such that:

iii. $d(x, y)=0$ if and only if $x=y$.

Then, $d$ it said to be a quasi-metric on $X$.

For a given quasi-(pseudo-)metric space $(X, d)$, the function $d^{-1}$ defined on $X \times X$ by $d^{-1}(x, y)=d(y, x)$ for all $x, y \in X$, is also a quasi-(pseudo-)metric on $X$ called the conjugate quasi-(pseudo-)metric of $d$, and the function $d^{s}$ defined on $X \times X$ by $d^{s}=\max \left\{d(x ; y), d^{-1}(x, y)\right\}$ for all $x, y \in X$ is a (pseudo-)metric on $X$.

Each extended quasi-pseudo-metric $d$ on $X$ generates a topology $\mathcal{T}_{d}$ on $X$ which has as a base the family of $d$-balls $\left\{B_{d}(x, r): x \in X, r>0\right\}$, where $B_{d}(x, r)=\{y \in X: d(x, y)<r\}$. Aiso, $d$ induces a quasi-uniformity $\mathcal{U}(d)$ on $X$ which has as a base the family $\left\{U_{n}: n \in \mathbb{N}\right\}$, where $U_{n}=\{(x, y) \in X \times X$ : $\left.d(x, y)<2^{-n}\right\}$ for all $n \in \mathbb{N}$.

A topological space $(X, \mathcal{T})$ is said to be quasi-pseudo-metrizable if there is a quasi-(pseudo-)metric $d$ on $X$ such that $\mathcal{T}=\mathcal{T}_{d}$. 
Let $(X, d)$ be an extended quasi-pseudo-metric space, for each $A, B \in \mathcal{P}_{0}(X)$ define

$$
\mathcal{H}_{d}(A, B)=\max \left\{\mathcal{H}_{d}^{-}(A, B), \mathcal{H}_{d}^{+}(A, B)\right\}
$$

where

$$
\mathcal{H}_{d}^{-}(A, B)=\sup _{a \in A} d(a, B) \text { and } \mathcal{H}_{d}^{+}(A, B)=\sup _{b \in B} d(A, b) .
$$

Then, (see for instance [8]), $H_{d}^{-}, H_{d}^{+}$and $\mathcal{H}_{d}$ are extended quasi-pseudo-metrics on $\mathcal{P}_{0}(X)$. Moreover, $\mathcal{H}_{d}$ is extended quasi-metric on $\mathcal{P}_{c l_{\cap}}(X)=\left\{c l_{\mathcal{T}_{d}}(A) \cap\right.$ $\left.c l_{\mathcal{T}_{d^{-1}}}(A) ; A \in \mathcal{P}_{0}(X)\right\} . H_{d}$ is called the Hausdorff quasi-metric of $d$.

\section{Probabilistic Quasi-Metric Space}

Recall ([9]) that a non-negative real function $f$ defined on $\mathbb{R}^{+} \cup\{+\infty\}$ is known as a distance distribution function (briefly, a d.d.f) if it is non-decreasing, left continuous on $] 0,+\infty[$, with $f(0)=0$ and $f(+\infty)=1$. The set of all d.d.f will be denoted by $\Delta^{+}$; and the set of all $f \in \Delta^{+}$for which $\lim _{s \rightarrow+\infty} f(s)=1$ by $D^{+}$.

A simple example of distribution function is Heavyside function in $D^{+}$, defined by

$$
H(t)=\left\{\begin{array}{lll}
0 & \text { if } & t=0 \\
1 & \text { if } & t>0
\end{array}\right.
$$

According to ([9]) A commutative, associative and nondecreasing mapping $\tau$ : $\Delta^{+} \times \Delta^{+} \rightarrow \Delta^{+}$is called a triangle function if and only if $\tau(f, H)=f$ for all $f \in \Delta^{+}$.

Definition 1. [9], [10] A triangle function $\tau$ is called sup-continuous if

$$
\tau\left(\sup _{i \in I} F_{i}, G\right)=\sup _{i \in I} \tau\left(F_{i}, G\right),
$$

for any family $\left\{F_{i}: i \in I\right\}$ of $\Delta^{+}$and any $G \in \Delta^{+}$.

Definition 2. ([2]) A probabilistic quasi-pseudo-metric space (briefly, PqpM space) is a triple $(M, F, \tau)$ where $M$ is a nonempty set, $F$ is a function from $M \times M$ into $\Delta^{+}, \tau$ is a triangle function and for all $p, q, r \in M$ the following conditions are satisfied:

i. $F_{p p}=H$,

ii. $F_{p, q} \geq \tau\left(F_{p, r}, F_{r, q}\right)$. 
$F$ is called a probabilistic quasi-pseudo-metric on $M$. if $F$ satisfies the additional condition is satisfied

iii. $F_{p q}=F_{q p}=H$ if and only if $p=q$.

Then, $(M, F, \tau)$ is called a probabilistic quasi-metric space (briefly, PqM space) and $F$ is called a probabilistic quasi-metric on $M$.

Moreover, if $F$ satisfies condition

iv. $F_{p q}=F_{q p}$.

Then, $(M, F, \tau)$ is called a probabilistic metric space (briefly, PM space).

If $(M, F, \tau)$ is a PqpM space and $G: X \times X \rightarrow \Delta^{+}$is defined by:

$$
G_{x y}=F_{y x} \quad \text { for all x, y } \in M
$$

Then the triple $(M, G, \tau)$ is also a PqpM space, $G$ is called the conjugate of $F$. Moreover, $\left(M, F^{s}, \tau\right)$ is a probabilistic (pseudo-)metric space, where $F_{p q}^{s}=$ $\min \left\{F_{p q}, G_{p q}\right\}$ for all $p, q \in M$.

Let $(M, F, \tau)$ be a PqpM space (see [2]). If $\tau$ is continuous, the collection $\left\{N_{x}(t): x \in M, t>0\right\}$ union $\emptyset$ form a base for a topology on $M$, where $N_{x}(t)=\left\{y \in M: F_{x y}(t)>1-t\right\}$. The topology determined in this way by $F$ will be denoted by $\mathcal{T}_{F}$. Similarly the conjugate $G$ of $F$ determined a topology $\mathcal{T}_{G}$. The family $\mathcal{U}(F)=\left\{U_{t}: t>0\right\}$ form a basis of a quasi-uniform structure on $M$ where $U_{t}$ is defined by $U_{t}=\left\{(x, y) \in M ; F_{x y}(t)>1-t\right\}$. Moreover, the conjugate quasi-uniformity $\mathcal{U}(F)^{-1}$ coincides with $\mathcal{U}(G)$ and it is compatible with $\mathcal{T}_{G}$.

Theorem 3. ([2]) If $(M, F, \tau)$ is a PqpM space and $\tau$ is continuous. Then $\mathcal{U}(F)$, or equivalently $\mathcal{T}_{F}$ is quasi-pseudo-metrizable.

Definition 4. ([2]) Let $(M, F, \tau)$ be a PqpM space:

1. A sequence $\left(x_{n}\right)_{n \in \mathbb{N}}$ in $M$ is said to be right $F$-convergent to $x \in M$, if for each $t>0$ there is $k \in \mathbb{N}$ such that $F_{x_{n} x}(t)>1-t$ whenever $n \geq k$.

2. A sequence $\left(x_{n}\right)_{n \in \mathbb{N}}$ in $M$ is said to be right K-Cauchy, if for each $t>0$ there is $k \in \mathbb{N}$ such that $F_{x_{n} x_{m}}(t)>1-t$ whenever $m \geq n \geq k$.

3. $(M, F, \tau)$ is said to be right $\mathrm{K}$-sequentially complete if every right $\mathrm{K}$ Cauchy sequence converge to some point in $M$. (with respect to the topology $\mathcal{T}_{F}$ ).

the proof of the following lemma is easy to reproduce so it is omitted. 
Lemma 5. Let $(M, F, \tau)$ be a PqpM space and let $d$ be a quasi-pseudometric on $M$ such that $\mathcal{U}(F)=\mathcal{U}(d)$ then we have

i. A sequence in $M$ is right $K$-Cauchy in $(M, F, \tau)$ if and only if it is right $K$-Cauchy in $(M, d)$.

ii. $(M, F, \tau)$ is right $K$-sequentially complete if and only if $(M, d)$ is right $K$ sequentially complete.

\section{Probabilistic Hausdorff Quasi-Metric}

In the sequel, when we speak about a PqpM space $(M, F, \tau)$, we always assume that $\tau$ is continuous. In this section we introduce and discuss the notion of the probabilistic Hausdorff quasi-(pseudo-)metric for a given probabilistic quasi(pseudo-)metric space on the sets $\mathcal{P}_{0}(M)$ and $\mathcal{P}_{c l_{\cap}}(M)$ where $\mathcal{P}_{c l_{\cap}}(M)$ is defined as in Section 1

$$
\mathcal{P}_{c l_{\cap}}(M)=\left\{c l_{\mathcal{T}_{F}}(A) \cap c l_{\mathcal{T}_{G}}(A) ; A \in \mathcal{P}_{0}(M)\right\}
$$

Note that for a PqpM space $(M, F, \tau)$ we have $\mathcal{P}_{c l_{\cap}}(M) \subset \mathcal{P}_{c l}(M) \subset \mathcal{P}_{0}(M)$, since for all $A \subset M$ we have $c l_{\mathcal{T}_{F}}(A)=\operatorname{cl}_{\mathcal{T}_{F}}\left(c_{\mathcal{T}_{F}}(A)\right) \cap c l_{\mathcal{T}_{G}}\left(c_{\mathcal{T}_{F}}(A)\right)$.

We begin by recalling the concept of the probabilistic Hausdorff distance and some knowing results.

Let $(M, F, \tau)$ be a PM space. Given $p \in M, B \in \mathcal{P}_{0}(M)$. "The probabilistic distance" form $p$ to $B$ is defined as

$$
F_{p B}(t)=F_{B p}(t)=\sup _{q \in B} F_{p q}(t) \quad \text { for all } t \in[0,+\infty]
$$

Given $A, B \in \mathcal{P}_{0}(M)$ and defined

$$
\mathcal{H}_{F}^{-}(A, B)(t)=\left\{\begin{array}{l}
0 \text { if } t=0 \\
\sup _{0<s<t} \inf _{p \in A} F_{p B}(s) \text { if } t \in(0,+\infty]
\end{array}\right.
$$

and

$$
\mathcal{H}_{F}^{+}(A, B)(t)=\left\{\begin{array}{l}
0 \text { if } t=0 \\
\sup _{0<s<t} \inf _{q \in B} F_{A q}(s) \text { if } t \in(0,+\infty]
\end{array}\right.
$$

The probabilistic Hausdorff distance between $A$ and $B$ is the d.d.f:

$$
\mathcal{H}_{F}(A, B)=\min \left\{\mathcal{H}_{F}^{-}(A, B), \mathcal{H}_{F}^{+}(A, B)\right\}
$$

Note that since $M$ is a $\mathrm{PM}$ space we have $\mathcal{H}_{F}^{+}(A, B)=\mathcal{H}_{F}^{-}(B, A)$. 
Theorem 6. ([9]) Let $(M, F, \tau)$ be PM space If the triangle function $\tau$ is sup-continuous then $\left(\mathcal{P}_{c l}, \mathcal{H}_{F}, \tau\right)$ is a PM space .

In the following lemmas we collect some properties which will be needed frequently.

Lemma 7. Let $(F, M, \tau)$ be PqpM space. Then for each $A, B \in \mathcal{P}_{0}(M)$ we have

i. $x \in \operatorname{cl}_{\mathcal{T}_{F}}(A)$ if and only if $F_{x A}=H$

ii. $A \subset \operatorname{cl}_{\mathcal{T}_{F}}(B)$ if and only if $\mathcal{H}_{F}^{-}(A, B)=H$

iii. $B \subset \operatorname{cl}_{\mathcal{T}_{G}}(A)$ if and only if $\mathcal{H}_{F}^{+}(A, B)=H$

Proof. $(i)$ let $x \in \operatorname{cl}_{\mathcal{T}_{F}}(A), t>0$ and $n \in \mathbb{N}$ such that $t>\frac{1}{n}$, then there exists $y_{n} \in A$ such that $F_{x y_{n}}\left(\frac{1}{n}\right)>1-\frac{1}{n}$. Then, $F_{x A}(t) \geq \sup _{a \in A} F_{x a}\left(\frac{1}{n}\right) \geq$ $F_{x y_{n}}\left(\frac{1}{n}\right)>1-\frac{1}{n}$, so by letting $n \rightarrow+\infty$ we obtain $F_{x A}(t)=1$. Next, suppose that $F_{x A}=H$. Let $t>0$, since sup $F_{x a}(t)=1$ there exist $y \in A$ such that $F_{x y}(t)>1-t$ then $y \in N_{x}(t) \cap A$.

(ii) Suppose that $A \subset c l_{\mathcal{T}_{F}}(B)$. Let $p \in A$ and $0<s<t$ then by $(i) F_{p B}(s)=1$, so $\inf _{p \in A} F_{p B}(s)=1$. Thus we have $\mathcal{H}_{F}^{-}(A, B)(t)=\sup _{0<s<t} \inf _{p \in A} F_{p B}(s)=1$

conversely. Let $t>0$ since $\sup _{0<s<t} \inf _{p \in A} F_{p B}(s)=1$ there exists a sequence $\left(s_{n}\right)_{n \in \mathbb{N}}$ with $s_{n} \in(0, t)$ for each $n \in \mathbb{N}$ and $\inf _{p \in A} F_{p B}\left(s_{n}\right)>1-\frac{1}{n}$. Now for each $a \in A$ we have $F_{a B}(t) \geq \inf _{p \in A} F_{p B}(t) \geq \inf _{p \in A} F_{p B}\left(s_{n}\right)>1-\frac{1}{n}$ for all $n \in \mathbb{N}$, then $F_{a B}(t)=1$, so $a \in c l_{\mathcal{T}_{F}}(B)$. Likewise we show $(i i i)$.

A slight modification of the proof of Theorem 4.13 of [10], permits us to show the following result.

Lemma 8. Let $(M, F, \tau)$ be a PqpM space. if $\tau$ is sup-continuous then for each $A, B, C \in \mathcal{P}_{0}(M)$ we have

i. $\mathcal{H}_{F}^{-}(A, B) \geq \tau\left(\mathcal{H}_{F}^{-}(A, C), \mathcal{H}_{F}^{-}(C, B)\right)$

ii. $\mathcal{H}_{F}^{+}(A, B) \geq \tau\left(\mathcal{H}_{F}^{+}(A, C), \mathcal{H}_{F}^{+}(C, B)\right)$

Now from the above lemmas we deduce the following result.

Theorem 9. Let $(M, F, \tau)$ be PqpM space such that that $\tau$ is supcontinuous then 
i. $\left(\mathcal{P}_{0}(M), \mathcal{H}_{F}^{-}, \tau\right),\left(\mathcal{P}_{0}(M), \mathcal{H}_{F}^{+}, \tau\right)$ and $\left(\mathcal{P}_{0}(M), \mathcal{H}_{F}, \tau\right)$ are a PqpM spaces.

ii. If in addition $(M, F, \tau)$ is a PqM space, then $\left(\mathcal{P}_{c l_{n}}(M), \mathcal{H}_{F}, \tau\right)$ is PqM space.

Proof. $i$. from Lemma 7, Lemma 8 and the definition of $\mathcal{H}_{F}^{-}, \mathcal{H}_{F}^{+}$and $\mathcal{H}_{F}$. it follows immediately that $\left(\mathcal{P}_{0}(M), \mathcal{H}_{F}^{-}, \tau\right),\left(\mathcal{P}_{0}(M), \mathcal{H}_{F}^{+}, \tau\right)$ and $\left(\mathcal{P}_{0}(M), \mathcal{H}_{F}, \tau\right)$ are a PqpM spaces.

ii. suppose that $(M, F, \tau)$ is a PqM space and let $G$ be the conjugate of $F$, since $\mathcal{P}_{c l_{\cap}}(M) \subset \mathcal{P}_{0}(M)$, then by $(i)\left(\mathcal{P}_{c l_{\cap}}(M), \mathcal{H}_{F}, \tau\right)$ is a PqpM space. Now Let $A, B \in \mathcal{P}_{c l_{\cap}}(M)$ such that $\mathcal{H}_{F}(A, B)=\mathcal{H}_{F}(B, A)=H$, then by Lemma 7 (i) we have $A \subset c l_{\mathcal{T}_{F}}(B)$ and $B \subset c l_{\mathcal{T}_{F}}(A)$, and by Lemma 7 (ii) we have $A \subset c l_{\mathcal{T}_{G}}(B)$ and $B \subset c l_{\mathcal{T}_{G}}(A)$. Thus $A \subset c l_{\mathcal{T}_{F}}(B) \cap c l_{\mathcal{T}_{G}}(B)=B$ and $B \subset$ $c l_{\mathcal{T}_{F}}(A) \cap c l_{\mathcal{T}_{G}}(A)=A$, so $A=B$. Then we conclude that $\left(\mathcal{P}_{c l_{\cap}}(M), \mathcal{H}_{F}, \tau\right)$ is PqM space.

\section{Properties of the Probabilistic Hausdorff Quasi-Metric}

Let $(X, \mathcal{U})$ be a quasi-uniform space, for a subsets $A$ of $X$ and $U \in \mathcal{U}$ define :

$$
U(A)=\bigcup_{x \in A} U(x)=\{y \in X:(x, y) \in U \text { for some } x \in A\}
$$

For each $U \in \mathcal{U}$ let

$$
\begin{gathered}
\bar{U}=\left\{(A, B) \in \mathcal{P}_{0}(X) \times \mathcal{P}_{0}(X): B \subseteq U(A)\right\}, \\
\underline{U}=\left\{(A, B) \in \mathcal{P}_{0}(X) \times \mathcal{P}_{0}(X): A \subseteq U^{-1}(B)\right\},
\end{gathered}
$$

and

$$
U^{\sim}=\left\{(A, B) \in \mathcal{P}_{0}(X) \times \mathcal{P}_{0}(X): B \subseteq U(A) \text { and } A \subseteq U^{-1}(B)\right\} .
$$

Then, (see for instance [6]), $\{\bar{U}: U \in \mathcal{U}\}$ form a base for a quasi-uniformity $\overline{\mathcal{U}}$ on $\mathcal{P}_{0}(X),\{\underline{U}: U \in \mathcal{U}\}$ form a base for quasi-uniformity $\underline{\mathcal{U}}$ on $\mathcal{P}_{0}(X)$ and $\left\{U^{\sim}: U \in \mathcal{U}\right\}$ form a base for a quasi-uniformity $\mathcal{U}^{\sim}$ on $\mathcal{P}_{0}(X)$ called the Bourbaki (or Hausdorff) quasi-uniformity induced by $\mathcal{U}$ on $\mathcal{P}_{0}(X)$.

Remark 10. Let $(X, d)$ be quasi-pseudo-metric space. Then

1. $\overline{\mathcal{U}(d)}=\mathcal{U}\left(\mathcal{H}_{d}^{+}\right), \underline{\mathcal{U}(d)}=\mathcal{U}\left(\mathcal{H}_{d}^{-}\right)$and $\mathcal{U}(d)^{\sim}=\mathcal{U}\left(\mathcal{H}_{d}\right)$ on $\mathcal{P}_{0}(M)$. (see [1]). 
2. $\left(\mathcal{P}_{0}(X), \mathcal{H}_{d}\right)$ is right $\mathrm{K}$-sequentially complete if and only if $(X, d)$ is right K-sequentially complete (see $[6],[8]$ ).

Theorem 11. Let $(M, F, \tau)$ be a PqpM space. If there is a quasi-pseudo metric $d$ such that $\mathcal{U}(F)=\mathcal{U}(d)$. Then $\mathcal{U}\left(\mathcal{H}_{F}\right)=\mathcal{U}\left(\mathcal{H}_{d}\right)$ on $\mathcal{P}_{0}(M)$.

Proof. Let $(M, F, \tau)$ be a PqpM space and suppose that there is a quasipseudo metric $d$ such that $\mathcal{U}(F)=\mathcal{U}(d)$ then from Remark 10 (1) we have $\mathcal{U}(F)^{\sim}=\mathcal{U}(d)^{\sim}=\mathcal{U}\left(\mathcal{H}_{d}\right)$, to conclude it remain to claim that $\mathcal{U}(F)^{\sim}=\mathcal{U}\left(\mathcal{H}_{F}\right)$. To this end we need to show that $\mathcal{U}\left(\mathcal{H}_{F}^{-}\right)=\mathcal{U}(F)$ and $\mathcal{U}\left(\mathcal{H}_{F}^{+}\right)=\overline{\mathcal{U}(F)}$. Let $t>0$, $0<s<t$ and $A, B \in \mathcal{P}_{0}(X)$ such that $A \subseteq \widetilde{C U}_{s}^{-1}(B)$, then for each $a \in A$ there exists $b \in B$ such that $F_{a b}(s)>1-s$, so we have $F_{a B}(s)>1-s>1-t$. Then we get $\inf _{a \in A} F_{a B}(s)>1-t$ and hence $\mathcal{H}_{F}^{-}(A, B)(t)>1-t$. Thus $\underline{\mathcal{U}(F)} \subseteq \mathcal{U}\left(\mathcal{H}_{F}^{-}\right)$ on $\mathcal{P}_{0}(M)$. Conversely, if for a $t>0$ we have $\mathcal{H}_{F}^{-}(A, B)(t)>1-t$, then there exists $0<s<t$ such that $F_{a B}(s)>1-t$ for all $a \in A$, so $A \subseteq U_{t}^{-1}(B)$, then $\mathcal{U}\left(\mathcal{H}_{F}^{-}\right) \subseteq \mathcal{U}(F)$. on $\mathcal{P}_{0}(M)$

Likewise we show that $\mathcal{U}\left(\mathcal{H}_{F}^{+}\right)=\overline{\mathcal{U}(F)}$ on $\mathcal{P}_{0}(M)$. hence $\mathcal{U}(F)^{\sim}=\mathcal{U}\left(\mathcal{H}_{F}\right)$ on $\mathcal{P}_{0}(M)$. Which complete the proof

Theorem 12. Let $(M, F, \tau)$ be a PqpM space such that $\tau$ is sup-continuous. Then

i. $(M, F, \tau)$ is right $K$-sequentially complete if and only if $\left(\mathcal{P}_{0}(M), \mathcal{H}_{F}, \tau\right)$ is right $K$-sequentially complete.

ii. If in addition $(M, F, \tau)$ is a PqM space. Then, $(M, F, \tau)$ is right $K$ sequentially complete if and only if $\left(\mathcal{P}_{c l_{\cap}}(M), \mathcal{H}_{F}, \tau\right)$ is right $K$-sequentially complete.

Proof. The proof of (i) follows immediately from Theorem 9, Theorem 11, Lemma 5 and Remark 10 (2).

Next, Let $(M, F, \tau)$ the given PqM space and $G$ the conjugate of $F$. Suppose That $\left(\mathcal{P}_{c l_{\cap}}(M), \mathcal{H}_{F}, \tau\right)$ is right $K$-sequentially complete and let $\left(x_{n}\right)_{n \in \mathbb{N}}$ be a right $K$-Cauchy sequence in $(M, F, \tau)$. At first observe that for each $n \in \mathbb{N}\left\{x_{n}\right\}=\operatorname{cl}_{\mathcal{T}_{F}}\left\{x_{n}\right\} \cap c l_{\mathcal{T}_{G}}\left\{x_{n}\right\}$. We can easily verify that $\left(C_{n}\right)_{n \in \mathbb{N}}$ is a right $K$-Cauchy sequence in $\mathcal{P}_{c l_{\cap}}(M)$ where $C_{n}=\left\{x_{n}\right\}$ for each $n \in \mathbb{N}$. Since $\left(\mathcal{P}_{c l_{\cap}}(M), \mathcal{H}_{F}, \tau\right)$ is right $K$-sequentially complete, there exists $C$ such that $C_{n} \rightarrow C$. it is easy to claim that $\left(x_{n}\right)_{n \in \mathbb{N}}$ converge to every $x \in C$. Hence we deduce $(M, F, \tau)$ is right $K$-sequentially complete.

Conversely, assume that $(M, F, \tau)$ is right $K$-sequentially complete. Let $\left(C_{n}\right)_{n \in \mathbb{N}}$ 
be a right K-Cauchy sequence in $\mathcal{P}_{c l_{\cap}}(M)$, then from (i) and since $\mathcal{P}_{c l_{\cap}}(M) \subset$ $\mathcal{P}_{0}(M)$ there exists $C \in \mathcal{P}_{0}(M)$ such that $\left(C_{n}\right)_{n \in \mathbb{N}}$ converge to $C$. We will claim that $\left(C_{n}\right)_{n \in \mathbb{N}}$ converge to $c_{\mathcal{T}_{F}}(C)$ in $\mathcal{P}_{c l_{n}}(M)$ with respect to $\mathcal{T}_{\mathcal{H}_{F}}$. At first observe that $\left(C_{n}\right)_{n \in \mathbb{N}}$ converge to $c l_{\mathcal{T}_{F}}(C)$ with respect to $\mathcal{T}_{\mathcal{H}_{F}^{+}}$since $C \subset c l_{\mathcal{J}_{F}}(C)$. Next, Let $t>0$ such that $U_{t} \subseteq \mathcal{U}(F), x \in c l_{\mathcal{T}_{F}}(C)$ and $0<s<t$, then there exists $c \in C$ such that $x \in U_{s}^{-1}(c)$ and since $C_{n} \rightarrow C$ there is $n_{0} \in \mathbb{N}$ such that $C \subseteq U_{s}^{-1}\left(C_{n}\right)$ whenever $n \geq n_{0}$. hence for each $n \geq n_{0}$ we have $x \in \bigcup_{c \in U_{s}^{-1}\left(C_{n}\right)} U_{s}^{-1}(c)=U_{s}^{-2}\left(C_{n}\right) \subset U_{t}^{-1}\left(C_{n}\right)$. Thus $\left(C_{n}\right)_{n \in \mathbb{N}}$ converge to $c l_{\mathcal{T}_{F}}(C)$ with respect to $\mathcal{T}_{\mathcal{H}_{F}^{-}}$. Since $c l_{\mathcal{T}_{F}}(C) \in \mathcal{P}_{c l_{\cap}}(M)$ we conclude that $\left(\mathcal{P}_{c l_{\cap}}(M), \mathcal{H}_{F}, \tau\right)$ is right $\mathrm{K}$-sequentially complete.

\section{References}

[1] G. Berthiaume, On quasi-uniformities in hyperspaces, Proc. Amer. Math. Soc., 66, No.2 (1977), 335-343.

[2] Y.J.Cho, M.Grabiec, V.Radu, On Nonsymmetric Topological and Probabilistic Structures, NovaSci.Publ., NewYork (2006).

[3] R.J. Egbert, Products and quotients of probabilistic metric spaces, Pacific J. Math., 24, (1968), 437-455.

[4] P. Fletcher, W.F. Lindgren, Quasi-Uniform Spaces, Marcel Dekker, New York, (1982).

[5] H.P.A.Künzi, Nonsymmetric topology, Bolyai Soc. Math. Stud., Topology, Szeksárd, Hungary (Budapest)., 4 (1993), 303-338.

[6] H.P.A.Künzi, C.Ryser, The Bourbaki quasi-uniformity, Topology Proc., 20, (1995), 161-183.

[7] K. Menger, Statistical metrics, Proc. Nat. Acad, of Sci., U.S.A., 28, (1942), 535-537.

[8] M.A.Sánchez-Granero, Weak completeness of the Bourbaki quasiuniformity, Appl. Gen.Topology., 2, No.1 (2001), 101-112.

[9] B. Schweizer and A.Sklar, Probabilistic Metric Spaces, Series in Probability and Applied Mathematics, North-Holland (1983). 
[10] R.M. Tardiff, Topologies for probabilistic metric spaces, Pacific J. Math., 65, No.1 (1976), 233-251. 